\title{
Search for Higgs bosons beyond the Standard Model
}

\author{
Rainer Mankel ${ }^{1, a}$, on behalf of the CMS Collaboration \\ ${ }^{1}$ Deutsches Elektronen-Synchrotron (DESY) \\ Notkestr 85, D-22603 Hamburg, Germany
}

\begin{abstract}
While the existence of a Higgs boson with a mass near $125 \mathrm{GeV}$ has been clearly established, the detailed structure of the entire Higgs sector is yet unclear. Beyond the standard model interpretation, various scenarios for extended Higgs sectors are being considered. Such options include the minimal and next-to-minimal supersymmetric extensions (MSSM and NMSSM) of the standard model, more generic Two-Higgs Doublet models (2HDM), as well as truly exotic Higgs bosons decaying e.g. into totally invisible final states. This article presents recent results from the CMS experiment.
\end{abstract}

\section{Introduction}

The discovery of a Higgs boson at a mass near $125 \mathrm{GeV}$ has been a milestone for Elementary Particle Physics $[1,2]$. One of the most relevant questions after this discovery concerns the structure of the Higgs sector. Up to now, the measured properties of the observed state are in accord with the expectations of the standard model (SM). Nevertheless, the observed particle could well be only the first member of an extended Higgs sector. Detailed analysis of the Higgs couplings as they are measured from the various decay channels indicate that there is still plenty of room for non-SM decays of the $\mathrm{H}(125)$ boson [3]. The most promising approach is the direct search for additional Higgs bosons, or for decays that are forbidden in the SM.

\section{Higgs Models Beyond the Standard Model}

Supersymmetry presents an elegant solution to the quadratic divergences in the Higgs mass radiative corrections, since the super-partners of the classical particles cancel the corresponding loop contributions. In order to achieve this cancellation, supersymmetry naturally requires additional Higgs bosons. The minimal sypersymmetric standard model (MSSM) features two complex Higgs doublets, leading to five physical Higgs boson states, three of them neutral (h, H, A), commonly denoted as $\phi$, and two of them charged $\left(\mathrm{H}^{ \pm}\right)$. At tree level, the MSSM is governed by two parameters, $\mathrm{m}_{\mathrm{A}}$ and $\tan \beta$, where the latter is the ratio between the vacuum expectation values of the two Higgs doublets.

The next-to minimal sypersymmetric standard model (NMSSM) is characterized by two complex Higgs doublets plus an additional singlet field. This results in seven physical Higgs states: $\mathrm{h}_{1}, \mathrm{~h}_{2}, \mathrm{~h}_{3}$, $\mathrm{a}_{1}, \mathrm{a}_{2}$ and $\mathrm{h}^{ \pm}$.

\footnotetext{
ae-mail: Rainer.Mankel@desy.de
} 
The two-Higgs doublet models (2HDM) can be seen as an effective theory, which extends the SM by adding a second complex Higgs doublet. Flavor conservation can be imposed by enforcing symmetries. This leads to four types of 2HDM - Type I, Type II, Lepton-specific and Flipped - which differ in the way the Higgs doublets couple to the fermions. The structure of the MSSM corresponds to a Type II 2HDM, with a similar set of Higgs bosons. In less restricted 2HDM, also flavor-changing Yukawa couplings are possible.

Other models are inspired by warped extra dimensions (WED), as the Randall-Sundrum model. Such models may predict new heavy particles that can decay into pairs of $\mathrm{H}(125)$ Higgs bosons. Examples are the radion, which is a particle with zero spin, and the first Kaluza-Klein excitation of the graviton, which has spin two.

\section{Search for Neutral MSSM Higgs Bosons in the $\tau \tau$ Channel}

The decay $\phi \rightarrow \tau \tau$ represents a good compromise between a relatively large expected branching fraction, and manageable backgrounds. Both leptonic $\left(\tau_{\mathrm{e}}, \tau_{\mu}\right)$ and hadronic $\tau$ decays $\left(\tau_{\text {had }}\right)$ are considered, and five of the six possible combinations $-\tau_{\mathrm{e}} \tau_{\mu}, \tau_{\mathrm{e}} \tau_{\text {had }}, \tau_{\mu} \tau_{\text {had }}, \tau_{\text {had }} \tau_{\text {had }}$ and $\tau_{\mu} \tau_{\mu}-$ are used in this analysis. The main production modes considered are gluon-gluon fusion, characterized by absence of any b-tagged jet, and b-associated production, where at least one b-tagged jet is required.

The main backgrounds and the way they are estimated in this analysis can only be addressed in broad strokes. Contributions from decays of the $\mathrm{Z}$ boson into the $\tau \tau$ final state are estimated using an embedding method. In this technique $\mathrm{Z} \rightarrow \mu \mu$ decays are extracted from real data, and the muon products are replaced by simulated $\tau$ decays. The $\mathrm{Z} \rightarrow \mu \mu$ background itself, which is most relevant for the $\tau_{\mathrm{e}} \tau_{\mu}$ and $\tau_{\mu} \tau_{\mu}$ channels, is suppressed by using the distance of closest approach of the two muon tracks. All distributions are found to be well described by the background hypothesis.
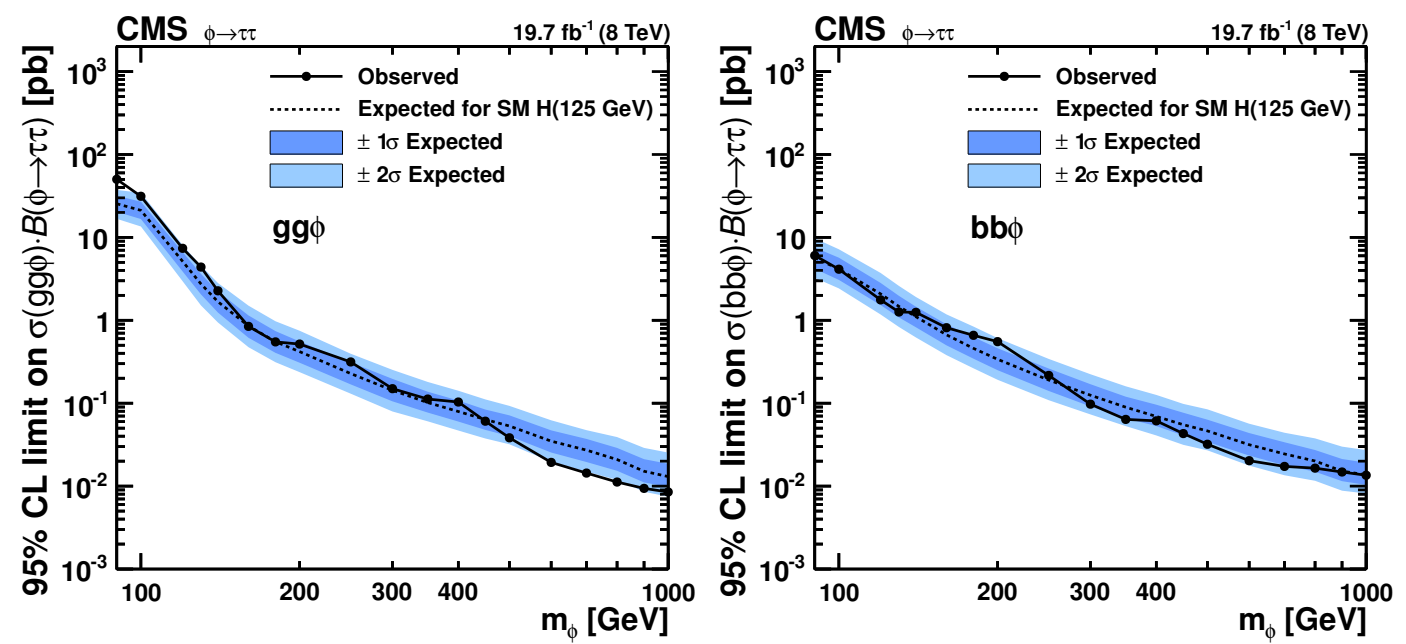

Figure 1. Upper limits on $\sigma \cdot \mathcal{B}(\phi \rightarrow \tau \tau)$ at $95 \% \mathrm{CL}$ for gluon fusion (left) and b-quark associated neutral Higgs boson production (right) for a single narrow resonance (right) [4].

In absence of a signal, upper limits are computed for $\sigma \cdot \mathcal{B}(\phi \rightarrow \tau \tau)$, separately for the gluon fusion and b-associated production modes, as they are shown in Figure 1. The observed and expected 
limits agree well, where the latter take the contribution of a SM Higgs boson at $125 \mathrm{GeV}$ into account. The combination of the 7 and $8 \mathrm{TeV}$ datasets allows extension of the Higgs mass range up to $1 \mathrm{TeV}$.
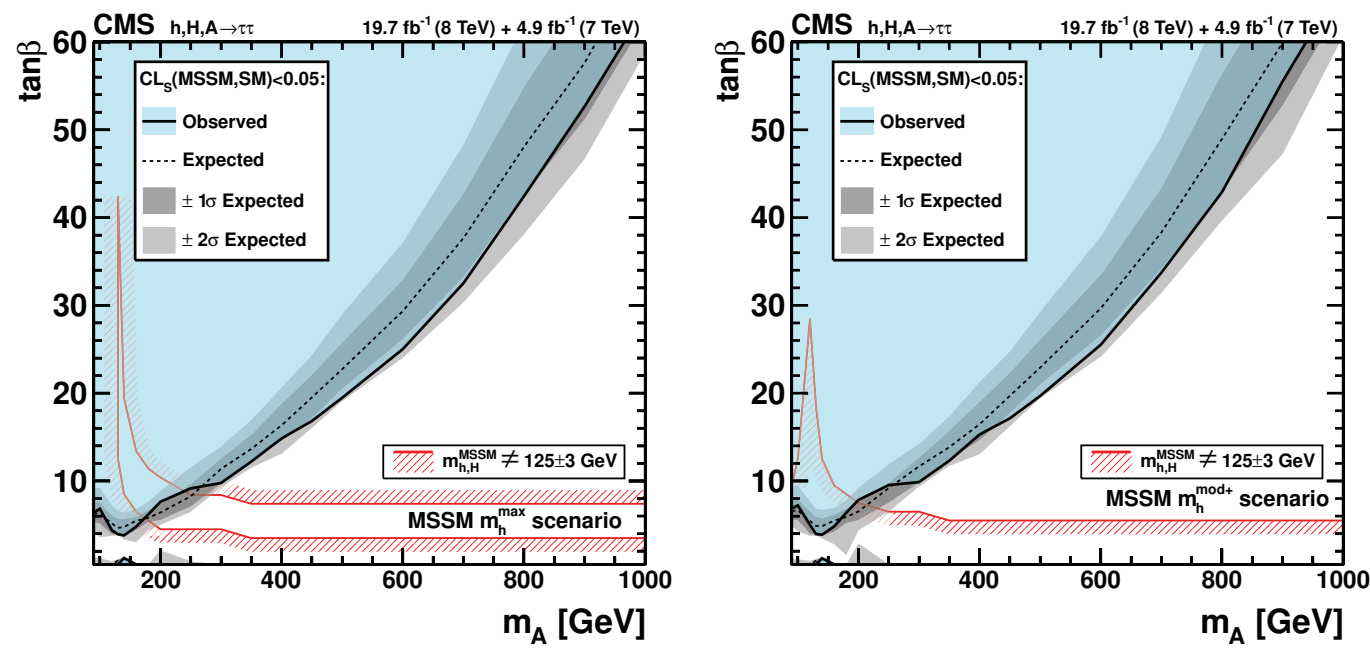

Figure 2. Expected and observed exclusion at 95\% CL limits in the $\mathrm{m}_{\mathrm{A}}-\tan \beta$ parameter space for the MSSM $\mathrm{m}_{\mathrm{h}}^{\max }$ and $\mathrm{m}_{\mathrm{h}}^{\text {modt+ }}$ benchmark scenarios [4].

The results are interpreted in the context of the MSSM. The full MSSM Higgs mass spectrum is taken into account. A test statistic comparing the likelihoods of the MSSM and SM hypotheses is computed for each MSSM parameter point, and a point is taken as excluded if the confidence level of the MSSM hypothesis is less than 5\%. The resulting constraints in the space of the MSSM parameters $\mathrm{m}_{\mathrm{A}}$ and $\tan \beta$ are shown in Figure 2. The left plot shows the exclusion limits in the traditional $\mathrm{m}_{h}^{\max }$ scenario. In this scenario, which was defined before the discovery of the boson at $125 \mathrm{GeV}$, the radiative correction parameters were tuned such that the mass of the lightest MSSM Higgs boson, h, became as large as possible. As a result, only a narrow band mostly at moderate values of $\tan \beta$ seems possible with the $m_{h}$ mass still compatible with the measurement at $125 \mathrm{GeV}$.

However, this band does not take any uncertainties of the MSSM parameters beyond the tree level into account. Recently, new benchmark scenarios have been created [5] which are better adjusted to the measured mass of the Higgs boson at $125 \mathrm{GeV}$. The right plot in Figure 2 shows the exclusion limits in the $\mathrm{m}_{\mathrm{h}}^{\bmod +}$ scenario. The $125 \mathrm{GeV}$ mass constraint only results in a lower constraint on $\tan \beta$, and the upper limits only come from the direct search.

\section{Charged Higgs Bosons}

Observation of a charged Higgs boson would be immediate evidence for physics beyond the SM. If the mass of the $\mathrm{H}^{ \pm}$is below the top mass, it can be produced in top decays. In the MSSM with $\tan \beta<1$, the dominant decay mode is $\mathrm{H}^{+} \rightarrow \mathrm{c} \overline{\mathrm{s}}$. If the second top quark in a $\bar{t} \overline{\mathrm{t}}$ event decays semi-leptonically, the event topology is similar to that of the t⿱亠t lepton-plus-jets channel, and the signal should appear as a second peak besides the $\mathrm{W}^{+}$in the di-jet invariant mass distribution.

The recent CMS analysis [6] performs a search for this channel in events with an isolated muon, a minimum of four jets with at least two b-tags, and a modest requirement of missing transverse energy 

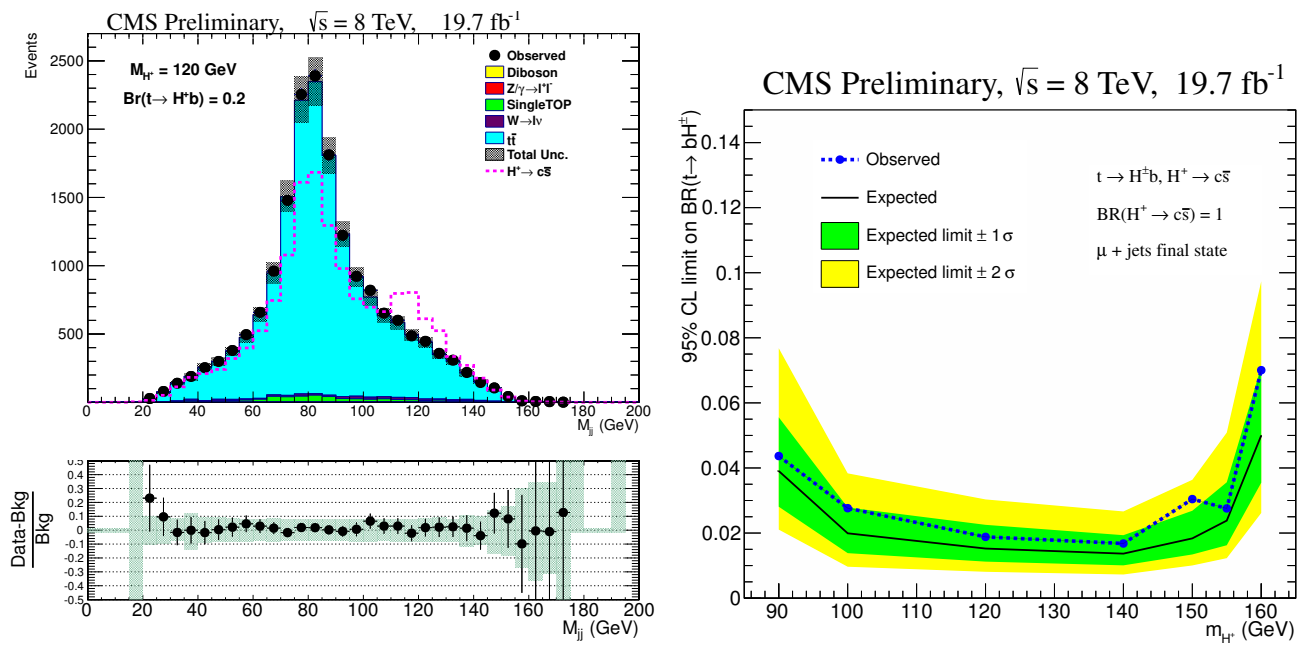

Figure 3. Left: Di-jet mass distributions after all selections. The dotted line represents the total expected event yield in the presence of a charged Higgs boson [6]. Right: Exclusion limit of $\mathcal{B}\left(\mathrm{t} \rightarrow \mathrm{H}^{+} \mathrm{b}\right)$ at $95 \%$ confidence level asuming $\mathcal{B}\left(\mathrm{H}^{+} \rightarrow \mathrm{cs}\right)=100 \%$.

to suppress QCD and Z+jet backgrounds. A kinematic fit is applied constraining both top candidates to their nominal mass, improving the mass resolution of the cs candidate. The resulting di-jet invariant mass distribution is shown in Figure 3 (left). This figure also shows the enhancement which would be expected if $20 \%$ of the top decays contained a $\mathrm{H}^{+}$boson with a mass of $120 \mathrm{GeV}$.

No such enhancement is observed in the data. The resulting upper limits for the branching fraction $\mathcal{B}\left(\mathrm{t} \rightarrow \mathrm{H}^{+} \mathrm{b}\right)$ ranges from $2-3 \%$ in the mass interval of $100-150 \mathrm{GeV}$, on the assumption of $\mathcal{B}\left(\mathrm{H}^{+} \rightarrow \mathrm{cs}\right)=100 \%$. These limits apply to practically any BSM resonance with the corresponding production and decay topology.

\section{Lepton-Flavor Violating Higgs Decays}

In the SM, decays violating the lepton flavor conservation are forbidden. However, such decays may be allowed in certain two-Higgs doublet models, composite Higgs or Randall-Sundrum models. This section focuses on a direct search for Higgs decays in the $\mathrm{H} \rightarrow \mu \tau$ channel [7]. The $\tau$ leptons are identified in their electronic $\left(\tau_{\mathrm{e}}\right)$ and hadronic $\tau_{\text {had }}$ decay modes. The signature is thus similar to the $\mathrm{H} \rightarrow \tau \tau$ analysis, but the kinematics differ strongly due to one neutrino less present in the final state. Events are selected which contain, besides an isolated muon, either an isolated lepton or a hadronic $\tau$ candidate. The events are categorized according to the number of jets. As a signal variable, the collinear mass is reconstructed from the visible decay products.

The analysis of the collinear mass spectra shows that the observed distributions are compatible with the background expectations. The observed upper limit for the branching fraction $\mathcal{B}(\mathrm{H} \rightarrow \mu \tau)$ is $1.57 \%$, compared to an expected limit of $0.75 \%$, at $95 \%$ confidence level. The best-fit result is $\mathcal{B}(\mathrm{H} \rightarrow \mu \tau)=\left(0.89_{-0.37}^{+0.40}\right) \%$. These results correspond to a mild excess of about 2.5 standard deviations, which is still in agreement with the SM. Figure 4 (left) shows the breakdown of the upper limit into the various event classes. 

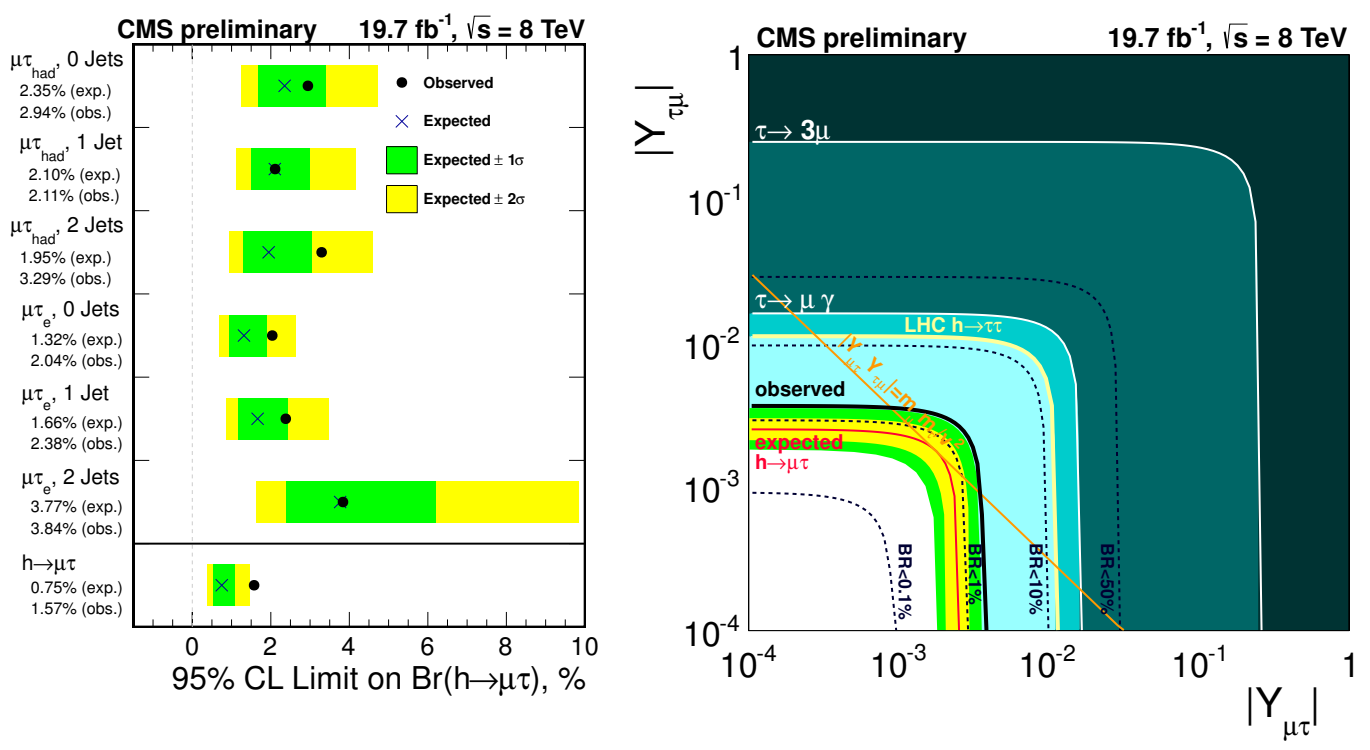

Figure 4. Left: Upper limits by category for lepton-flavor violating $\mathrm{H} \rightarrow \mu \tau$ decays [7]. Right: Constraints in the plane of the flavor-violating Yukawa couplings $\mathrm{Y}_{\tau \mu}$ and $\mathrm{Y}_{\mu \tau}$.

The result can be directly interpreted in terms of the anomalous Yukawa couplings $\mathrm{Y}_{\tau \mu}$ and $\mathrm{Y}_{\mu \tau}$, and the resulting constraint in the space of these two parameters is shown in Figure 4 (right). This new result improves on the best existing indirect measurements by a factor of $\approx 4.4$, leading to the best limits to date.

\section{Resonant Production of Higgs Pairs}

In the SM, the rate of Higgs pair production is very small, and beyond experimental reach with the currently available integrated luminosity recorded at the LHC. But heavy resonances decaying into Higgs pairs in the context of BSM physics could have a much higher cross section, which could be probed with the existing dataset. Candidates for such resonances are heavy MSSM or NMSSM Higgs bosons decaying into a pair of $\mathrm{H}(125)$ bosons, but also radions or Kaluza-Klein excitations of the graviton in the context of warped extra dimensions.

The analysis presented here [8] combines the H(125) decay channels into b $\bar{b}$ and $\gamma \gamma$, which have the virtues of large branching fraction and good mass resolution, respectively. The selections are similar to the corresponding SM inclusive analyses. A mass-constraint fit is performed on the $b \bar{b}$ candidate using the known mass of the $\mathrm{H}(125)$, which improves significantly the mass resolution of the resonance candidates. The signal is searched for in the $\mathrm{m}_{\gamma \gamma}$ distribution for low $\mathrm{m}_{\mathrm{X}}(<400 \mathrm{GeV})$ and in the $\mathrm{m}_{\gamma \gamma \mathrm{bb}}$ distribution in the high $\mathrm{m}_{\mathrm{X}}$ region. Beyond $1100 \mathrm{GeV}$, there is an increased probability for the b $\bar{b}$ pair to merge into a single "fat" jet; for this reason, the search is not continued beyond this point. Medium and high purity selections are performed depending on whether b-tagging is required on one or both legs of the di-jet candidate. Even the medium purity selection is characterized by low QCD background because the presence of two photons is required. Selected invariant mass spectra are shown in Figure 5. They do not exhibit any significant peak, only a smooth background structure. 

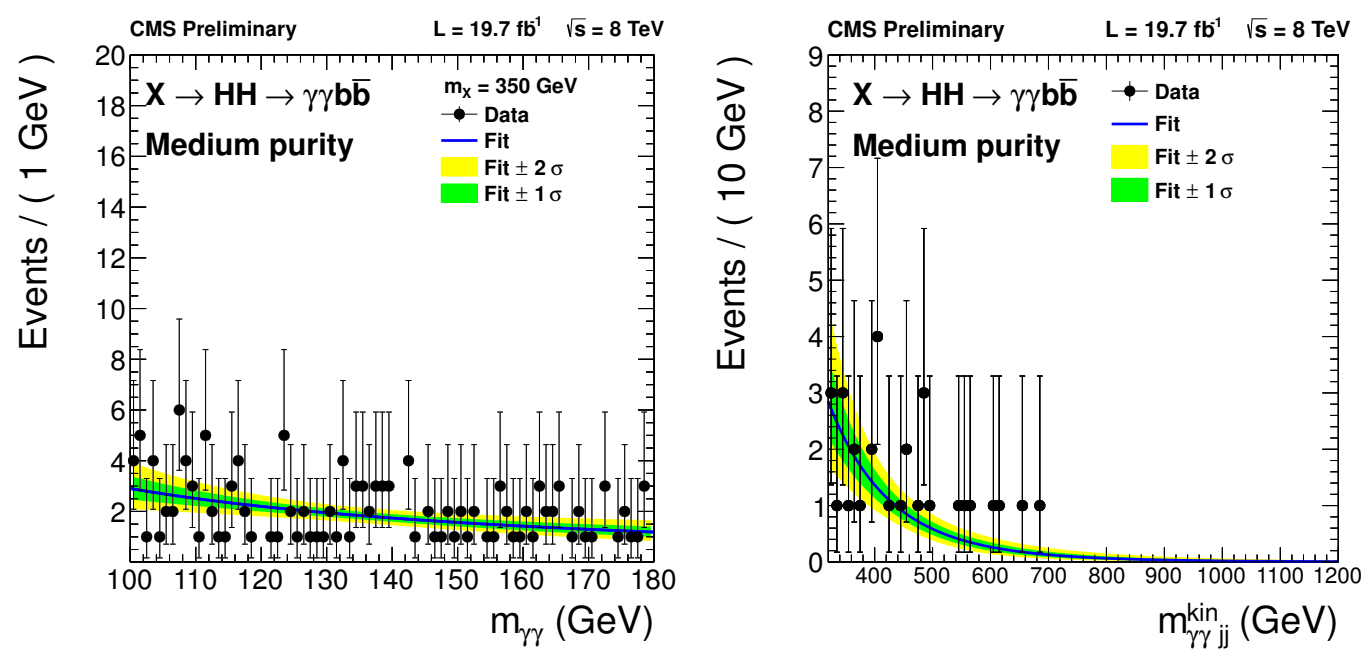

Figure 5. Left: Di-photon invariant mass spectrum from the low- $m_{X}$ analysis for a mass hypothesis of $\mathrm{m}_{\mathrm{X}}=350 \mathrm{GeV}$ [8]. Right: Spectrum of the invariant mass of the $\mathrm{HH}$ candidate in the high- $\mathrm{m}_{\mathrm{X}}$ region.

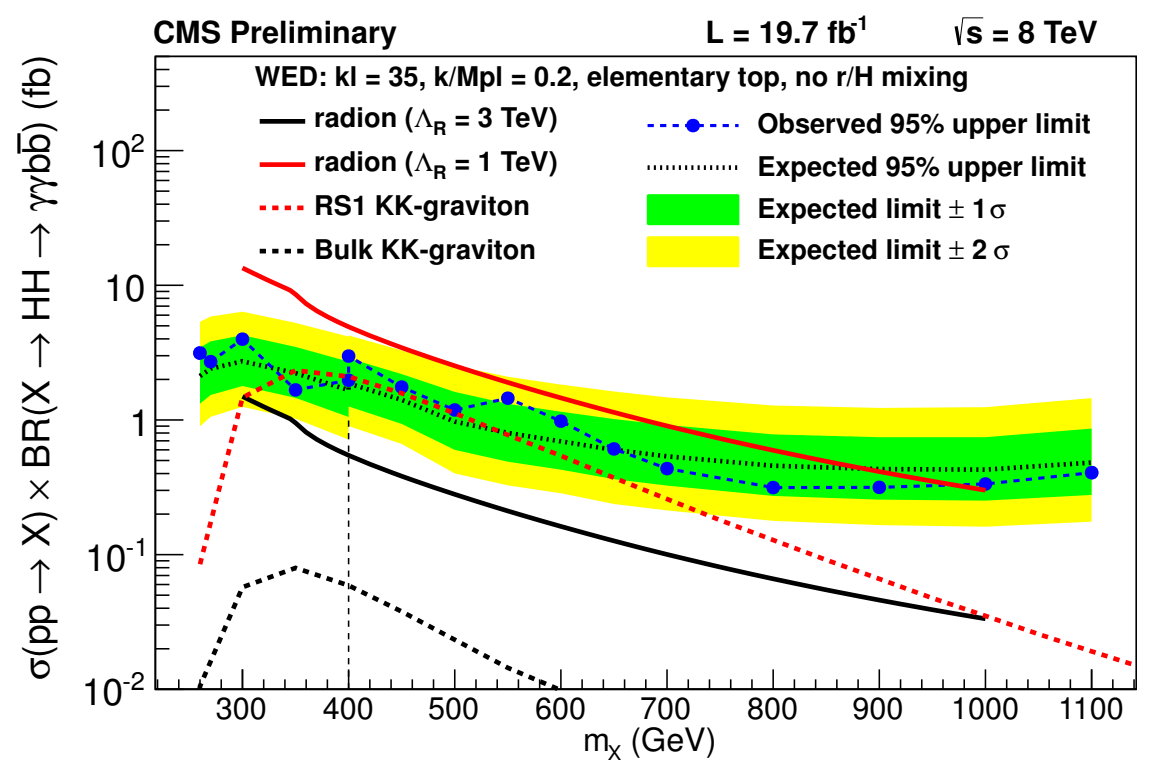

Figure 6. Expected and observed upper limits on the cross section times branching fractions $\sigma(\mathrm{pp} \rightarrow \mathrm{X}) \times$ $\mathcal{B}(\mathrm{X} \rightarrow \mathrm{HH} \rightarrow \gamma \gamma \mathrm{b} \overline{\mathrm{b}})$. Theory lines corresponding to WED models with radion, RS1 KK-graviton and bulk KKgraviton are also shown. The vertical dashed line shows the separation between the low and high mass analysis regions. 
In absence of a signal, upper limits on the product of cross section and branching fraction are derived and displayed in Figure 6. They are compared to predictions of radion and graviton models. The results exclude radions with $\mathrm{m}<970 \mathrm{GeV}$ for the radion scale $\Lambda_{\mathrm{R}}=1 \mathrm{TeV}$, and RS1 KK gravitons in the mass range of $340-400 \mathrm{GeV}$.

\section{Invisible Higgs Decays}

Invisible Higgs boson decays are possible in a wide range of models, including decays to neutralinos in supersymmetric models, or graviscalars in models with extra dimensions. According to Higgsportal models, the Higgs boson could be a mediator between the SM particles and particles of the dark matter sector. The analysis of couplings, under certain assumptions, constrains the branching fraction into non-SM decay modes indirectly to $<32 \%$ [3].
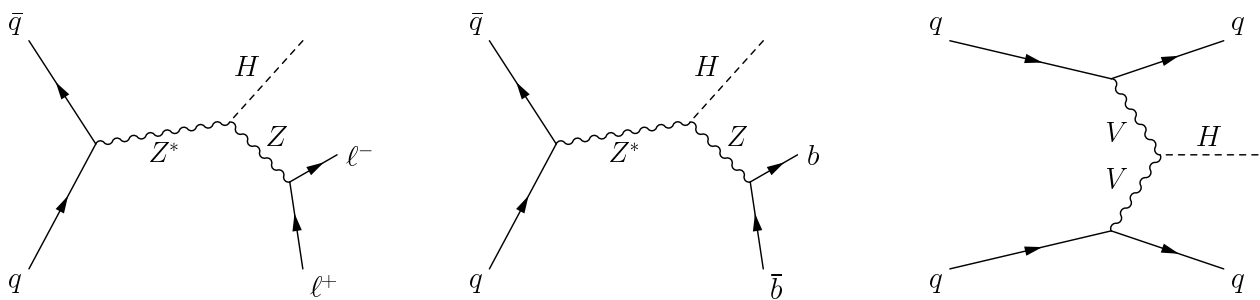

Figure 7. Feynman diagrams for Higgs production is association with a $\mathrm{Z}$ boson decaying into a pair of leptons (left) and b-quarks (middle), and in vector boson fusion (right). The Higgs boson is assumed to decay invisibly.

Direct searches for invisible Higgs decays are possible in final states where the Higgs boson recoils against a visible system [9]. Examples are the associated production with a $\mathrm{Z}$ boson decaying either leptonically or to a b-quark pair, as illustrated in Figure 7 (left) and (middle), or vector boson fusion (VBF) as depicted in Figure 7 (right).

While the VBF process is favored by the larger SM cross section, the final state of two jets plus missing transverse energy suffers from large backgrounds. These are mainly events containing vector bosons and additional jets, where the vector bosons are not seen, like $\mathrm{Z}$ bosons decaying into neutrinos. The signal is analyzed in the variables of missing transverse energy and di-jet mass. The observed distributions of these variables are shown in Figure 8. They are well described by the SM background distributions as they are estimated from data, and no excess attributable to invisible Higgs production is seen. The combined analysis of the VBF and Z-associated processes results in upper limits of the invisible branching fraction of a Higgs produced if it is produced according to the SM production cross section, which is shown in Figure 9. The observed upper limit of this branching fraction is 58\% at a mass of $125 \mathrm{GeV}$ at $95 \%$ confidence level. This result represents a considerable improvement with respect to earlier direct searches.

\section{Summary}

This article summarizes several recent analyses that address the fundamental question whether the observed Higgs boson at $\approx 125 \mathrm{GeV}$ could be just one member of an extended Higgs sector, and thus potentially a window into New Physics. While a sizable number of key signatures has been studied, only part of the range of possibilities has been constrained so far. The upcoming Run II of the LHC 

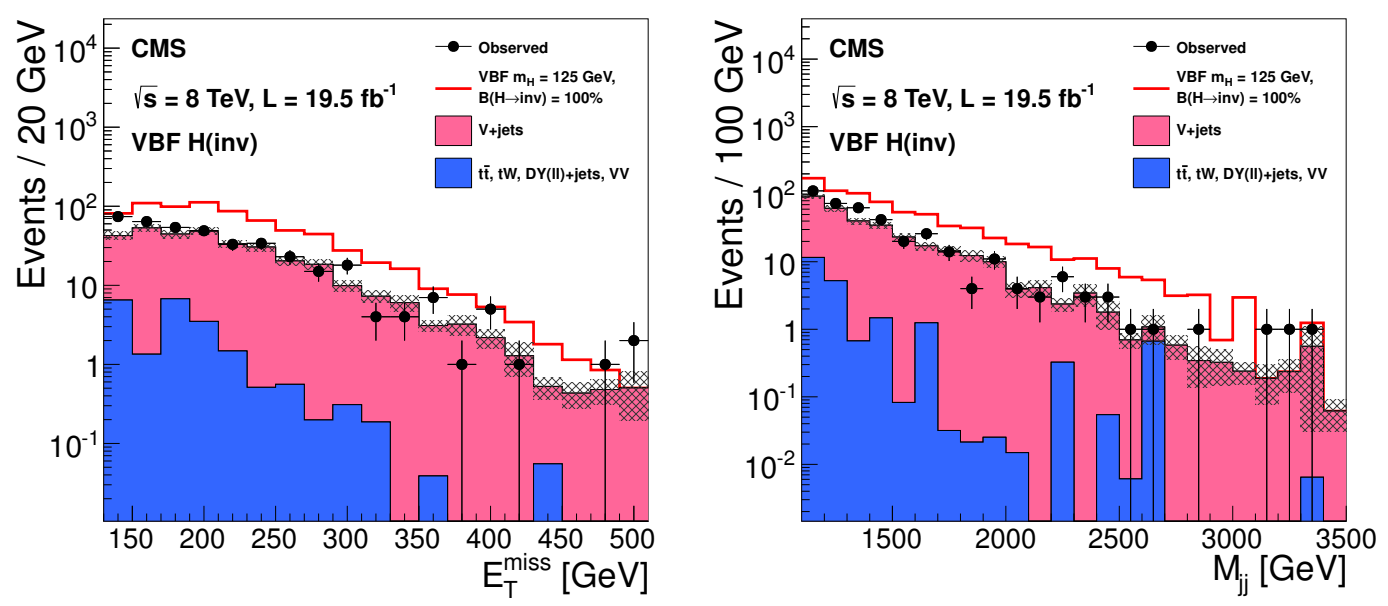

Figure 8. Distributions of missing transverse energy (left) and di-jet invariant mass (right) in the signal region of the invisible Higgs VBF analysis. A potential signal contribution from a hypothetical Higgs boson at a mass of $125 \mathrm{GeV}$ decaying exclusively into invisible modes is shown as red lines [9].

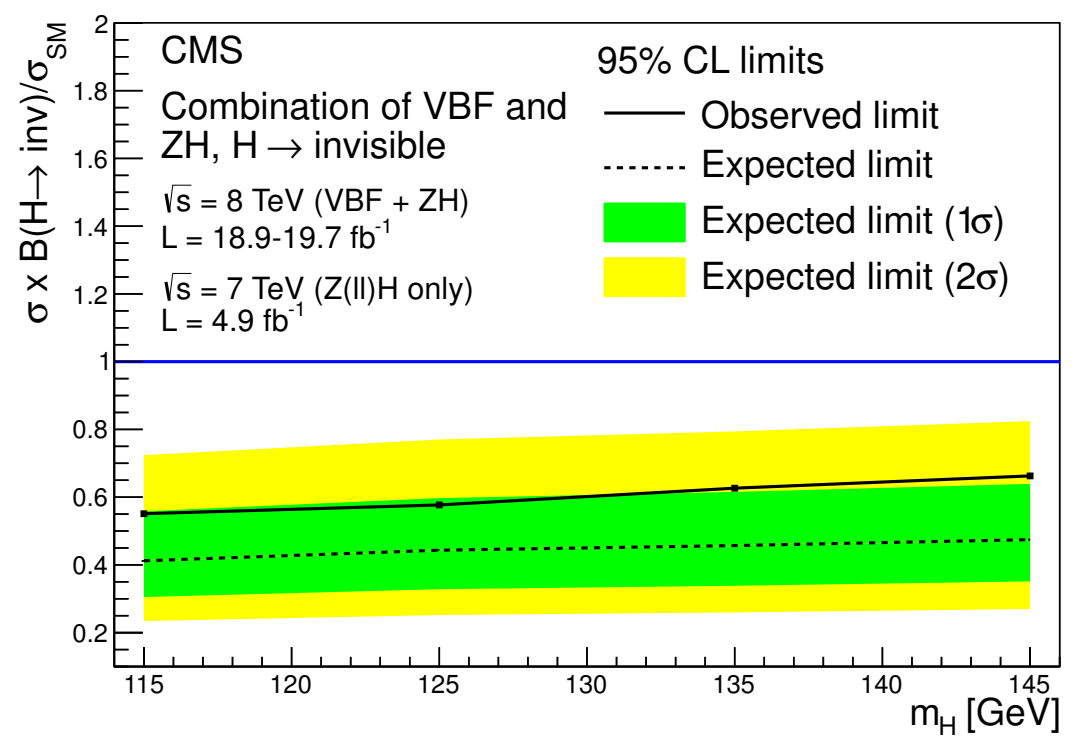

Figure 9. Expected and observed upper limits on $\sigma \cdot \mathcal{B}(\mathrm{H} \rightarrow$ inv $) / \sigma(\mathrm{SM})$ at $95 \%$ confidence level [9]. 
will significantly extend the reach, especially towards higher masses, and open a rich future research programme in the BSM Higgs sector.

\section{References}

[1] CMS Collaboration, "Observation of a new boson at a mass of $125 \mathrm{GeV}$ with the CMS experiment at the LHC”, Phys.Lett. B716 (2012) 3061.

[2] ATLAS Collaboration, "Observation of a new particle in the search for the Standard Model Higgs boson with the ATLAS detector at the LHC", Phys.Lett. B716 (2012) 129.

[3] CMS Collaboration, "Precise determination of the mass of the Higgs boson and studies of the compatibility of its couplings with the standard model", CMS PAS HIG-14-009 (2014).

[4] CMS Collaboration, "Search for neutral MSSM Higgs bosons decaying to a pair of tau leptons in pp collisions", JHEP 10 (2014) 160.

[5] M. Carena et al., "MSSM Higgs Boson Searches at the LHC: Benchmark Scenarios after the Discovery of a Higgs-like Particle", Eur.Phys.J. C73 (2013) 2552.

[6] CMS Collaboration, "Search for a light charged Higgs boson in the $\mathrm{H}^{+} \rightarrow c \bar{s}$ channel", CMS PAS HIG-13-035 (2013).

[7] CMS Collaboration, "Search for lepton flavor violating decays of the Higgs boson", CMS PAS HIG-14-005 (2014).

[8] CMS Collaboration, "Search for the resonant production of two Higgs bosons in the final state with two photons and two bottom quarks", CMS PAS HIG-13-032 (2014).

[9] CMS Collaboration, "Search for invisible decays of Higgs bosons in the vector boson fusion and associated ZH production modes”, Eur.Phys.J. C74 (2014) 2980. 